\title{
Carriage of Supernumerary Sex Chromosomes Decreases the Volume and Alters the Shape of Limbic Structures
}

\author{
DiD Ajay Nadig, ${ }^{1}$ Paul K. Reardon, ${ }^{1}$ Jakob Seidlitz, ${ }^{1}$ Cassidy L. McDermott, ${ }^{1}$ Jonathan D. Blumenthal, ${ }^{1}$ Liv \\ S. Clasen, ${ }^{1}$ Francois Lalonde, ${ }^{1}$ Jason P. Lerch, ${ }^{2,3}$ M. Mallar Chakravarty, ${ }^{4,5}$ and ${ }^{10}$ Armin Raznahan ${ }^{1}$
}

https://doi.org/10.1523/ENEURO.0265-18.2018

${ }^{1}$ Developmental Neurogenomics Unit, Human Genetics Branch, National Institute of Mental Health, Bethesda, Maryland 20892, ${ }^{2}$ Department of Medical Biophysics, University of Toronto, Toronto, Ontario M5T 1R8, Canada, ${ }^{3}$ Neurosciences and Mental Health, the Hospital for Sick Children, Toronto, Ontario M5T 3H7, Canada, ${ }^{4}$ Cerebral Imaging Centre, Douglas Mental Health University Institute, McGill University, Montreal, Quebec H3A OG4, Canada,

${ }^{5}$ Department of Psychiatry, McGill University, Montreal, Quebec H3A OG4, Canada

\begin{abstract}
Sex chromosome aneuploidy (SCA) increases risk for several psychiatric disorders associated with the limbic system, including mood and autism spectrum disorders. Thus, SCA offers a genetics-first model for understanding the biological basis of psychopathology. Additionally, the sex-biased prevalence of many psychiatric disorders could potentially reflect sex chromosome dosage effects on brain development. To clarify how limbic anatomy varies across sex and sex chromosome complement, we characterized amygdala and hippocampus structure in a uniquely large sample of patients carrying supernumerary sex chromosomes $(n=132)$ and typically developing controls $(n=166)$. After adjustment for sex-differences in brain size, karyotypically normal males (XY) and females $(X X)$ did not differ in volume or shape of either structure. In contrast, all SCAs were associated with lowered amygdala volume relative to gonadally-matched controls. This effect was robust to three different methods for total brain volume adjustment, including an allometric analysis that derived normative scaling rules for these structures in a separate, typically developing population $(n=79)$. Hippocampal volume was insensitive to SCA after adjustment for total brain volume. However, surface-based analysis revealed that SCA, regardless of specific karyotype, was consistently associated with a spatially specific pattern of focal contraction and expansion in both amygdala and hippocampus. These results demonstrate the power of SCA as a model to understand how copy number variation can precipitate changes in brain systems relevant to psychiatric disease.
\end{abstract}

Key words: amygdala; CNV; hippocampus; sex chromosome aneuploidy

\section{Significance Statement}

In $\sim 1$ per 500 live births, infants are born carrying extra $X$ - or $Y$-chromosomes. These conditions, known as sex chromosome aneuploidies (SCA), are associated with elevated risk for psychiatric disorders such as depression, anxiety, and autism. In our study, we leverage a uniquely large dataset of brain scans from SCA patients to characterize how SCA influences the structure of the amygdala and hippocampus, - two structures which have been strongly implicated in many SCA-linked neuropsychiatric disorders. Across our different patient groups, we find converging evidence that SCA is associated with a decrease in amygdala volume, and spatially specific areal contractions in both the amygdala and hippocampus. Our findings help clarify how genetic copy number variation associated with psychiatric disease shapes brain organization. 
Table 1. Participant characteristics

\begin{tabular}{|c|c|c|c|c|c|c|c|c|}
\hline \multirow[b]{2}{*}{ Group } & \multicolumn{6}{|c|}{ Participant characteristics } & \multirow{3}{*}{$\begin{array}{l}X X X X Y \\
5\end{array}$} & \multirow{3}{*}{$\begin{array}{l}\text { Allometric } \\
\text { sample } \\
79 \text { (34 F) }\end{array}$} \\
\hline & $X X$ & $X Y$ & $X X X$ & $X X Y$ & $X Y Y$ & $X X Y Y$ & & \\
\hline $\begin{array}{l}n \\
\text { Age, y }\end{array}$ & 87 & 79 & 28 & 56 & 25 & 19 & & \\
\hline Mean (SD) & $12.7(5.1)$ & $12.7(4.6)$ & $12.3(5.7)$ & $12.7(4.9)$ & $12.2(4.9)$ & $14.0(5.5)$ & $12.9(4.8)$ & $13.0(0.6)$ \\
\hline $\begin{array}{l}\text { Range } \\
\text { FSIQ }\end{array}$ & $5.4-25$ & $5.2-25$ & $5.0-24.8$ & $5.2-26.0$ & $5.7-23.0$ & $5.0-23.0$ & $7.7-17.2$ & $12.0-14.0$ \\
\hline $\begin{array}{l}\text { Mean (SD)* } \\
\text { SES }\end{array}$ & $113.9(12.3)$ & $116.6(13.3)$ & $94.1(14.2)$ & $97.6(17.1)$ & $91.0(14.6)$ & 86.9 (12.9) & $55.7(7.23)$ & $116.3(12.4)$ \\
\hline Mean (SD)* & $47.3(17.1)$ & $48.0(21.3)$ & 40.9 (15.9) & $56.0(20.7)$ & $58.1(22.4)$ & $45.5(22.6)$ & $68.8(22.4)$ & $34.1(19.7)$ \\
\hline
\end{tabular}

FSIQ, Full scale IQ; SES, socioeconomic status.

${ }^{*} p<0.01$ for omnibus test of significant variation across groups in core sample.

\section{Introduction}

The amygdala and hippocampus are key elements of a limbic brain network that is perturbed in diverse neuropsychiatric disorders, including mood disorders (Price and Drevets, 2012) and autism (Amaral et al., 2008; Hennessey et al., 2018). Patients with sex chromosome aneuploidies (SCA) are at elevated risk for these psychiatric disorders (Hong and Reiss, 2014), although it remains unclear whether amygdalar and hippocampal development are sensitive to the presence of supernumerary sex chromosomes. Some evidence that the volume and shape of these structures may be sensitive to SCA comes from recent studies of Turner syndrome, which report that loss of one X-chromosome in females enlarges the amygdala in a spatially specific manner (Kesler et al., 2004; Green et al., 2016). However, it remains unknown whether these effects generalize to the hippocampus (the other core element of the limbic brain network), or whether either of these structures are sensitive to carriage of excess $\mathrm{X}$ - and or $\mathrm{Y}$-chromosomes. There are no existent studies of $\mathrm{Y}$-chromosome dosage effects on amygdala and hippocampus structure. Clarifying the nature of SCA effects on amygdalar and hippocampal anatomy is not only relevant for the neurobiology of sex chromosome aneuploidies, but also informs the broader question of sex-differences in amygdalar and hippocam-

Received July 10, 2018; accepted September 24, 2018; First published October 19, 2018.

The authors declare no competing financial interests.

Author contributions: A.N. and A.R. designed research; A.N., C.L.M., J.D.B., L.S.C., F.L., and A.R. performed research; A.N., P.K.R., J.S., and A.R. analyzed data; A.N. and A.R. wrote the paper; J.P.L. and M.M.C. contributed unpublished reagents/analytic tools.

This work was supported by the intramural program of the National Institutes of Health (Clinical trial NCT00001246, clinicaltrials.gov; NIH Annual Report Number, ZIA MH002794-13). P.K.R. and J.S. are supported by the NIH OxfordCambridge Scholars' Program. A.N. and C.S. are supported by NIH Intramural Research Training Awards. We thank the patients and their families for their participation in this study.

Correspondence should be addressed to Dr. Armin Raznahan, National Institute of Mental Health, Bethesda, Maryland 20892. E-mail: raznahana@mail.nih.gov.

https://doi.org/10.1523/ENEURO.0265-18.2018

Copyright (C) 2018 Nadig et al.

This is an open-access article distributed under the terms of the Creative Commons Attribution 4.0 International license, which permits unrestricted use, distribution and reproduction in any medium provided that the original work is properly attributed. pal anatomy. The fact that many disorders linked to limbic dysfunction also show clear sex-biases in prevalence (anxiety, $1.8 \times$ risk for females vs males: Ruscio et al., 2017; autism, $4 \times$ risk for males vs females: Werling and Geschwind, 2013) could potentially reflect sensitivity of limbic structures to biological factors that differ between males and females, such as $\mathrm{X}$ - and $\mathrm{Y}$-chromosome dosage.

Here, we detail sex and SCA effects on anatomy of the amygdala and hippocampus by applying recently developed methods for morphometric analysis (Chakravarty et al., 2013) to a large dataset of structural MRI scans from individuals with varying sex chromosome dosage $(n=298: 87 \mathrm{XX}, 79 \mathrm{XY}, 28 \mathrm{XXX}, 56 \mathrm{XXY}, 25 \mathrm{XYY}, 19$ $X X Y Y, 5 X X X X Y)$. This dataset has previously been used to profile sex and sex chromosome dosage effects on cortical, striatal, thalamic and cerebellar morphology (Lin et al., 2015; Fish et al., 2016; Raznahan et al., 2016; Reardon et al., 2016; Mankiw et al., 2017). A key methodological consideration in pursuing this goal is that sex chromosome aneuploidies are known to induce significant shifts in total brain volume (Raznahan et al., 2016), which may bias estimation of regional volumes given known patterns of non-linear subcortical scaling (Reardon et al., 2016, 2018). Therefore, we use a separate, typically developing sample $(N=79)$ to derive normative allometric scaling rules for the amygdala and hippocampus, and assess patient/control volume contrasts in the context of these scaling norms. Last, we determine whether changes in volume are accompanied by changes in the shape of limbic structures by mapping sex and SCA effects on local surface area across 5245 points (vertices) across the amygdalo-hippocampal surface. Moving beyond analysis of bulk volume to study shape is critical given the complex topography of functional and connectional gradients within the amygdala and hippocampus (Carr et al., 2010; Janak and Tye, 2015; Saygin et al., 2015).

\section{Materials and Methods}

\section{Sample}

Our core sample included 298 individuals of varying karyotype. Our allometric sample, used to generate scaling rules for the amygdala and hippocampus, included 79 typically developing $X X$ and $X Y$ individuals. Sample characteristics are described in Table 1. Participants with SCA were recruited through the National Institutes of Health $(\mathrm{NIH})$ 
website and parent support groups; $X-Y$-supernumeracy was confirmed by karyotype test. Non-mosaicism was confirmed by visualization of 50 metaphase spreads in peripheral blood. We excluded participants with a history of head injury, or gross brain abnormality. Typically developing individuals were drawn from the $\mathrm{NIH}$ Longitudinal Structural MRI Study of Human Brain Development (Giedd et al., 2015). Exclusion criteria for these participants included use of psychiatric medication, enrollment in special education services, history of mental health treatment, or prior diagnosis of a nervous system disorder. A $\chi^{2}$ test of independence revealed no significant differences in pubertal status (clinicianrated Tanner stage) between karyotype groups $\left(\chi^{2}=13.01\right.$, $p=0.966)$.

\section{Image acquisition and processing}

We collected T1-weighted MRI scans for each individual on the same 1.5 Tesla General Electric SIGNA scanner with contiguous $1.5 \mathrm{~mm}$ axial slices using a 3D spoiled gradient-recalled echo sequence with the following acquisition parameters: echo time, $5 \mathrm{~ms}$; repetition time, $24 \mathrm{~ms}$; flip angle, $45^{\circ}$; acquisition matrix, $256 \times 192$; number of excitations, 1 ; field-of-view, $24 \mathrm{~cm}$.

We estimated total brain volume by submitting scans to the CIVET 1.1.10 pipeline for automated morphometric analysis (Ad-Dab'bagh et al., 2006); these estimates have been reported in past studies (Raznahan et al., 2016; Reardon et al., 2016; Mankiw et al., 2017). This total brain volume estimate was the sum of total gray and whitematter volume estimates $\left(\mathrm{mm}^{3}\right)$, which were computed by CIVET tissue segmentation that used a validated neural net approach to voxel classification (Zijdenbos et al., 2002; Cocosco et al., 2003).

All amygdala and hippocampus measurements were automatically generated by a well validated multi-atlas segmentation algorithm, MAGeT Brain (Chakravarty et al., 2013; Pipitone et al., 2014). Briefly, this algorithm makes use of in vivo atlases generated high resolution T1- and T2-weighted images (3T, final isotropic voxel dimension $0.3 \mathrm{~mm}$ ) from five reference subjects ( 3 male, 2 female; Winterburn et al., 2013) that are aligned to 21 randomly selected participants within the NIH Human Brain Development in Health Study (Giedd et al., 2015) as previously described (Reardon et al., 2018). This pipeline produces a study-specific library of 105 amygdala and hippocampus segmentations (5 atlases $\times 21$ templates). The final segmentation is decided on using a voxel majority vote, where the most frequently applied label at each location is retained. These methods are reliable (Dice $\kappa=0.86$ ). A quality control image file is provided for each scan, allowing detailed visual inspection to rule out gross segmentation errors. All scans included in this analysis passed quality control of raw scans and MAGeT Brain segmentations for motion artifacts and other confounders by a rater who was blinded to subject karyotype (P.K.R.)

To determine shape, surface-based representation of amygdala and hippocampus structures were estimated using the marching cubes algorithm and morphologically smoothed using the AMIRA software package (Visage Imaging). Next, the nonlinear portions of the 21 transfor- mations mapping subjects to the 21 input templates were concatenated and averaged across input templates to limit the effects of noise and error. These surface-based representations were warped to fit each template, and each surface was warped to match each subject. This procedure yields 30 possible surface representations per subject that are then merged by estimating the median coordinate representation at each location. At this point, one-third of the surface of each triangle is assigned to each vertex within the triangle. The surface area value stored at each vertex is the sum of all such assignments form all connected triangles. Last, surface-area values were blurred with a surface-based diffusion-smoothing kernel $(5 \mathrm{~mm})$. The final derived measures of interest were total volume estimates for the left and right amygdala and hippocampus, and surface area at 5245 vertices across all structures (right amygdala: 1405; left amygdala: 1473; right hippocampus: 1215; left hippocampus: 1152).

\section{Statistical analyses}

\section{Bulk volume comparisons}

We first quantified the effect of karyotype on total bilateral amygdala and hippocampus volume in the core sample using omnibus $F$ tests. For structures showing a significant omnibus effect of group, follow-up post hoc $t$ tests were used to specify pairwise karyotype group differences in structure volume. To simultaneously visualize changes in regional and total brain volumes across karyotype groups, we plotted volumetric differences as an effect-size shift relative to the distribution observed in $X Y$ males. Effect sizes were calculated as (mean volume of $X Y$ group - mean volume of comparison group)/SD of $X Y$ group.

\section{Normative allometry of the amygdala and hippocampus}

We used a classic log-log regression approach (Huxley, 1924) to derive normative scaling rules for the amygdala and hippocampus within the independent allometric sample of 79 typically developing males and females. The scaling relationship between a regional volume and total brain volume is given by the $\beta_{1}$ coefficient in the following equation:

$\log _{10}($ Region volume $)=\beta_{0}+\beta_{1} \log _{10}($ Total brain volume $)+$ error.

This method allows simple quantification of non-linear allometric scaling relationships between regional and total brain volume. Furthermore, the scaling coefficient from this fit is easily interpretable: $\beta_{1}$ values significantly $<1$ indicate a hypoallometric scaling relationship (i.e., regional volume becomes proportionally smaller as total brain volume becomes larger); $\beta_{1}$ values significantly $>1$ indicate a hyperallometric scaling relationship (i.e., regional volume becomes proportionally larger as total brain volume becomes larger); $\beta_{1}$ values equal to 1 indicate an isometric scaling relationship (proportional size of the region is maintained across the range of total brain volume).

To account for sex-differences in the allometric fit $\beta_{0}$ and $\beta_{1}$ that have been observed in other brain structures (Reardon et al., 2016; Mankiw et al., 2017), we used our log-log regression framework to sequentially test three general linear models of amygdala and hippocampus allometry with decreasing complexity of sex differences. 
First, we tested for sex differences in the scaling coefficient itself, such that different allometric scaling principles hold for males and females $\left(\beta_{3}\right)$ :

$$
\begin{gathered}
\log _{10}\left(\text { Volume }_{\text {Re gion }}\right)=\beta_{0}+\beta_{1} \log _{10}\left(\text { Volume }_{\text {Total brain }}\right)+ \\
\beta_{2}(\text { Sex })+\beta_{3}\left(\log _{10}\left(\text { Volume }_{\text {Total brain }}\right) \times \text { Sex }\right)+\text { error. }
\end{gathered}
$$

In the absence of evidence for a sex difference in scaling $(p>0.05)$, we proceeded to the next model, which includes a single scaling relationship that allows for baseline sex difference in regional volume, reflected in the $\beta_{2}$ term:

$\log _{10}\left(\right.$ Volume $\left._{\text {Region }}\right)=\beta_{0}+\beta_{1} \log _{10}\left(\right.$ Volume $_{\text {Total brain }}+$ $\beta_{2}($ Sex $)+$ error.

In the absence of evidence for a sex difference in volume $(p>0.05)$, we proceeded to the last model, the simple allometric model with no sex term:

$\log _{10}\left(\right.$ Volume $\left._{\text {Region }}\right)=\beta_{0}+\beta_{1} \log _{10}\left(\right.$ Volume $\left._{\text {Total brain }}\right)+$ error.

\section{Sex and SCA effects in the context of allometry}

We used the scaling rules derived above to test for brain size-independent effects of sex chromosome complement on amygdala and hippocampus volumes. For each SCA group in our core sample (excluding the small sample of $5 \mathrm{XXXXY}$ individuals), we tested for differences between observed regional volumes and regional volumes predicted from total brain volume. For each regional volume, we selected the appropriate allometric model using the procedure defined above, and predicted regional volume for each individual in the core sample based on total brain volume and sex (including sex only if a significant sex difference in normative scaling was observed). We then calculated the deviation of each individual's observed $\log _{10}\left(\right.$ Volume $\left._{\text {Region }}\right)$ from the predicted $\log _{10}\left(V_{\text {Volume }}\right.$ Region $)$ with simple subtraction. Finally, we compared the distribution of these residuals between each SCA group and its appropriate gonadal control within the core sample (i.e., XXX vs XX, and other SCAs vs $X Y)$ using a regression approach with the following formula:

Deviation in $\log _{10}($ Region volume $)=\beta_{0}+\beta_{1}$ (Group) + error, where Group was a binary categorical variable. For each region, $p$ values from these tests were Bonferroni corrected to account for the five SCA group contrasts being performed.

Analysis of sex and SCA effects using normalization and covariation to control for brain size

We also compared the results of our allometric approach with two complementary approaches that are commonly used in the neuroanatomical literature: normalization and covariation. In normalization, group differences are tested after re-expressing regional volume as a fraction of total brain volume. In covariation, group differences are tested with total brain volume included as a covariate.

Normalization: Volume $_{\text {Region }}$ Volume $_{\text {Total brain }}=\beta_{0}+$ $\beta_{1}$ (Group) + error,

Covariation: Volume $_{\text {Region }}=\beta_{0}+\beta_{1}$ (Group) + $\beta_{2}\left(\right.$ Volume $\left._{\text {Total brain }}\right)+$ error.
To assess SCA effects, we ran these two models for each SCA group and its respective gonadal control group, such that Group was a binary categorical variable. Similar to our allometric approach, we Bonferroni corrected $p$ values for each region to account for the five contrasts being performed. To assess sex effects, we ran these models in our allometric sample, with Group reflecting sex.

All statistical analyses described above were performed using $R$ software (R Core Team, 2008). Data were visualized using the ggplot2 (Wickham, 2016) and patchwork (Pendersen, 2017) packages.

\section{Vertex-wise normative allometry of the amygdala and hippocampus surface}

To assess whether carriage of supernumerary sex chromosomes distorts amygdalar and hippocampal shape, we developed normative models for the relationship between shape and size of these structures. Because the MAGeT Brain morphometric pipeline represents structure shape as vertex-wise measures of local surface area, relationships between structure size and shape can be captured by modeling interindividual variation in area at each vertex by interindividual in total structure surface area, which is strongly related to total structure volume (Pearson's correlation between total region volume and total region surface areas: 0.97, amygdala; 0.89, hippocampus). We used a tiered approach to modeling sex differences similar to our bulk volume analyses. Critically, although vertex-wise variation in significance of sex differences is possible, for the purposes of simplicity and comparability of model parameters across vertices, we required that all vertices use the same final model. Thus, in our normative allometric sample $(n=79)$, we first modeled each vertex surface area estimate using the following equation:

$\log _{10}\left(\right.$ Surface area $\left.a_{\text {vertex }}\right)=\beta_{0}+\beta_{1} \log _{10}($ Total bilateral regional surface area) $+\beta_{2}($ Sex $)+$

$\beta_{3}\left[\log _{10}(\right.$ Total bilateral regional surface area $) \times$ Sex $]+$ error.

If any vertices contained significant interaction terms $\left(p_{\text {FDR }}<0.05\right)$, we applied this model to every vertex. In the absence of any vertices containing significant interaction terms, we proceeded to the next lower order vertex-wise model:

$\log _{10}\left(\right.$ Surface area $\left.a_{\text {vertex }}\right)=\beta_{0}+\beta_{1} \log _{10}($ Total bilateral regional surface area) $+\beta_{2}($ Sex $)+$ error.

If any vertices contained significant sex terms $\left(p_{\mathrm{FDR}}<0.05\right)$, we applied this model to every vertex. Finally, in the absence of any vertices containing significant sex terms, we proceeded to the lowest order, simple allometric vertex-wise model:

$\log _{10}\left(\right.$ Surface area $\left.a_{\text {vertex }}\right)=\beta_{0}+\beta_{1} \log _{10}($ Total bilateral regional surface area) + error.

Vertex-wise $\beta_{1}$ coefficients were visualized to represent which subregions scale hypo-, iso-, or hyperallometrically with total regional surface area.

Vertex-wise changes in surface area across SCA in the context of normative surface allometry

We performed a similar adjustment procedure as was applied in our volumetric analysis to assess allometrically adjusted changes in surface area across karyotype 
Table 2. Descriptive statistics for bulk volume analysis

\begin{tabular}{|c|c|c|c|c|c|c|c|c|c|}
\hline $\begin{array}{l}\text { Karyotype }(n) \\
\text { Total brain volume, } \mathbf{c m}^{3}\end{array}$ & \multicolumn{5}{|c|}{ Descriptive Statistics by Karyotype Group } & $X X Y Y(19)$ & $X X X X Y(5)$ & \multicolumn{2}{|c|}{$\begin{array}{l}\text { Omnibus test for } \\
\text { group differences }\end{array}$} \\
\hline SEM & 9.74 & 13.8 & 22.8 & 17.8 & 24.9 & 29.7 & 36.1 & & \\
\hline Amygdala, $\mathrm{cm}^{3}$ & & & & & & & & 23.78 & 22.15 \\
\hline Mean & 2.32 & 2.54 & 2.05 & 2.26 & 2.43 & 2.19 & 1.86 & & \\
\hline Mean & 4.30 & 4.57 & 3.98 & 4.32 & 4.68 & 4.33 & 3.82 & & \\
\hline SEM & 0.04 & 0.05 & 0.07 & 0.07 & 0.12 & 0.12 & 0.13 & & \\
\hline
\end{tabular}

groups. For each vertex, we predicted $\log _{10}\left(\right.$ Surface $\left._{\text {vertex }}\right)$, for each individual in the core sample based on total regional surface area and sex. We then calculated the deviation of each individual's actual $\log _{10}\left(\right.$ Surface $_{\text {vertex }}$ ) from the predicted $\log _{10}\left(\right.$ Surface $\left._{\text {vertex }}\right)$ at each vertex with simple subtraction. To assess SCA, we applied a simple model at each vertex separately for each contrast:

Deviation in $\log _{10}\left(\right.$ Surface $\left._{\text {vertex }}\right)=\beta_{0}+\beta_{1}($ Group $)+$ error,

where Group was a binary categorical variable. We corrected $p$ values separately for each contrast, for each region, using the FDR with $q$ (expected proportion of falsely-rejected nulls) set at 0.05 .

\section{Results}

Total brain volume, raw amygdala volume, and raw hippocampus volume are patterned across sex and sex chromosome complement

Total brain volume, amygdala volume, and hippocampus volume showed statistically significant variation across the seven distinct karyotype groups represented in our core sample of 298 individuals (Table 2; Fig. 1A). XX females had significantly smaller total brain, raw amygdala, and raw hippocampus volumes than $X Y$ males. Analysis of SCA revealed that carriage of a supernumerary X-chromosome was associated with decreased amygdala volume compared to gonadal controls. These differences were statistically-significant for all SCAs, and increased in effect size with mounting sex chromosome dosage. Carriage of a supernumerary X-chromosome was associated with decreased hippocampus volume compared to gonadal matched controls, except in the case of XXYY. In the small $(n=19)$ XXYY karyotype, we observed a non-significant reduction in hippocampus volume that was trend-level before correction for multiple comparisons $\left(p_{\mathrm{un}^{-}}\right.$ corrected $=0.08$ ), although this karyotype group was associated with the greatest effect size reduction in hippocampal volume. The XYY karyotype was not associated with significant differences in total brain volume, raw amygdala volume, or raw hippocampus volume as compared to $X Y$ males.

\section{The amygdala and hippocampus scale hypoallometrically with total brain volume}

In our allometric sample of 79 typically developing individuals, there were no significant effects of sex on either the intercepts or slopes of allometric models for amygdala or hippocampal volume (see Materials and Methods), so we proceeded with the simplest allometric model: $\log _{10}\left(\right.$ Volume $\left._{\text {Region }}\right)=\beta_{0}+\beta_{1} \log _{10}\left(\right.$ Volume $\left._{\text {Total brain }}\right)$. Both amygdala and hippocampus volume scaled hypoallometrically with total brain volume (Fig.1B; amygdala: $\beta_{1}=0.89$; hippocampus: $\beta_{1}=0.76$ ).

\section{A subset of sex and sex chromosome complement effects on amygdala and hippocampus volume survive allometric adjustment}

To assess whether changes in raw amygdala and hippocampus volume as a function of sex and SCA represent distortions of normative brain proportionality, we examined deviations from derived normative scaling rules across sex and sex chromosome complement (Fig. 1C). Sex differences in amygdala and hippocampus volume were not statistically-significant after allometric adjustment for total brain volume. The decrease in amygdala volume with carriage of a supernumerary X-chromosome remained significant after allometric adjustment. Allometric adjustment revealed a statistically-significant amygdala volume decrease in XYY versus $X Y$ males, where no significant change was detected in raw volumes. These results indicate a convergent effect of supernumerary $X$ and $\mathrm{Y}$-chromosome carriage on proportional amygdala volume. Differences in hippocampus volume across sex and sex chromosome complement were eliminated by allometric adjustment, indicating that proportional hippocampus volume is maintained across karyotype groups.

Results from normalization and covariation analysis were largely consistent with those of allometric analysis, with two notable exceptions. A significant decrease of hippocampus volume in males versus females was detected by normalization analysis, where an opposite effect was detected in raw volumes, and no significant effect was detected by covariation or allometric analysis. Also, decrease in amygdala volume in $X X X$ versus $X X$ groups was not significant in normalization analysis, but was significant in all other comparison techniques.

This pattern of results was robust to covariation for age and Tanner stage; a figure showing statistics for these comparisons is available online via FigShare (Nadig, 2018a). Notably, three XY participants, one XX participant, and one $X X Y$ participant included in the main analysis 
A

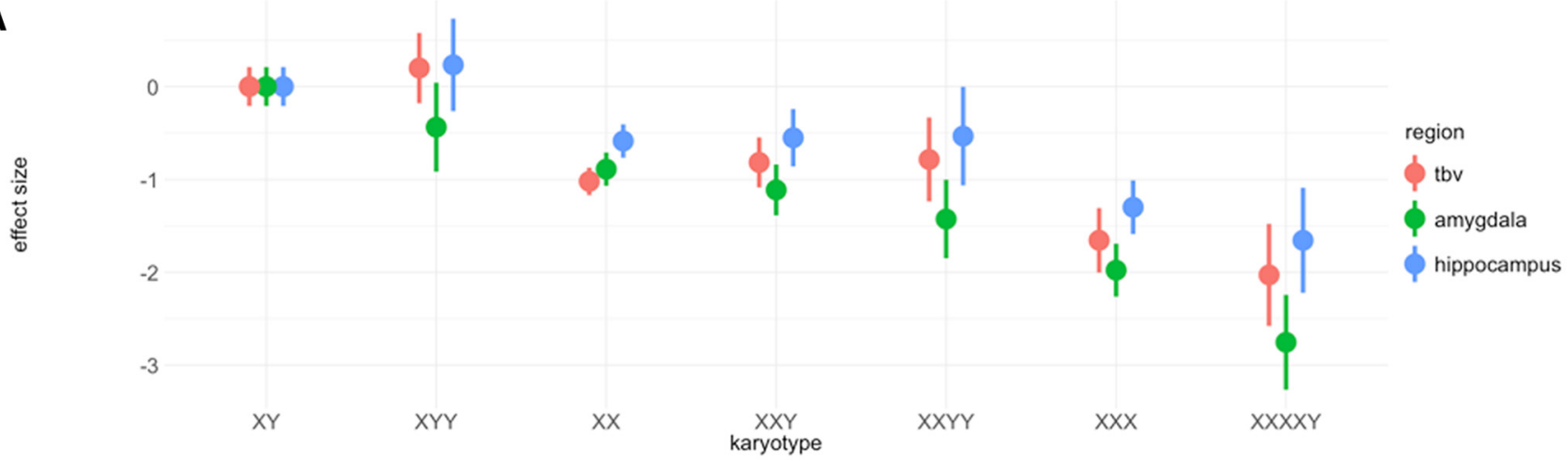

B

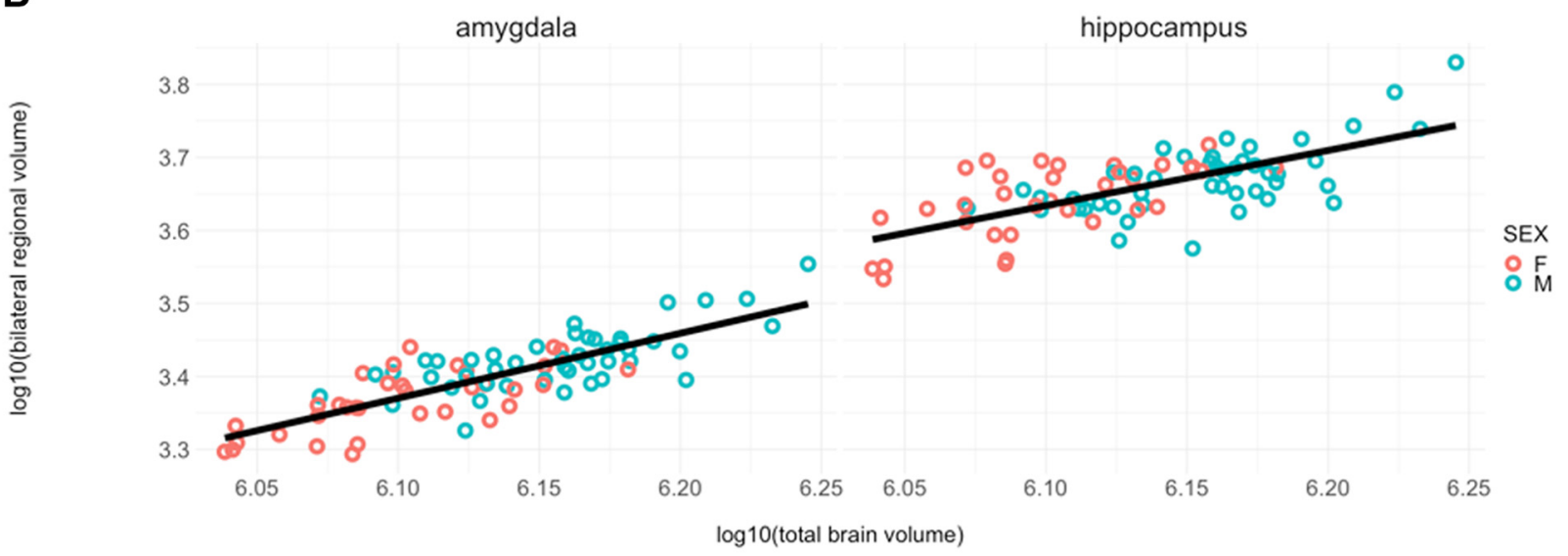

C
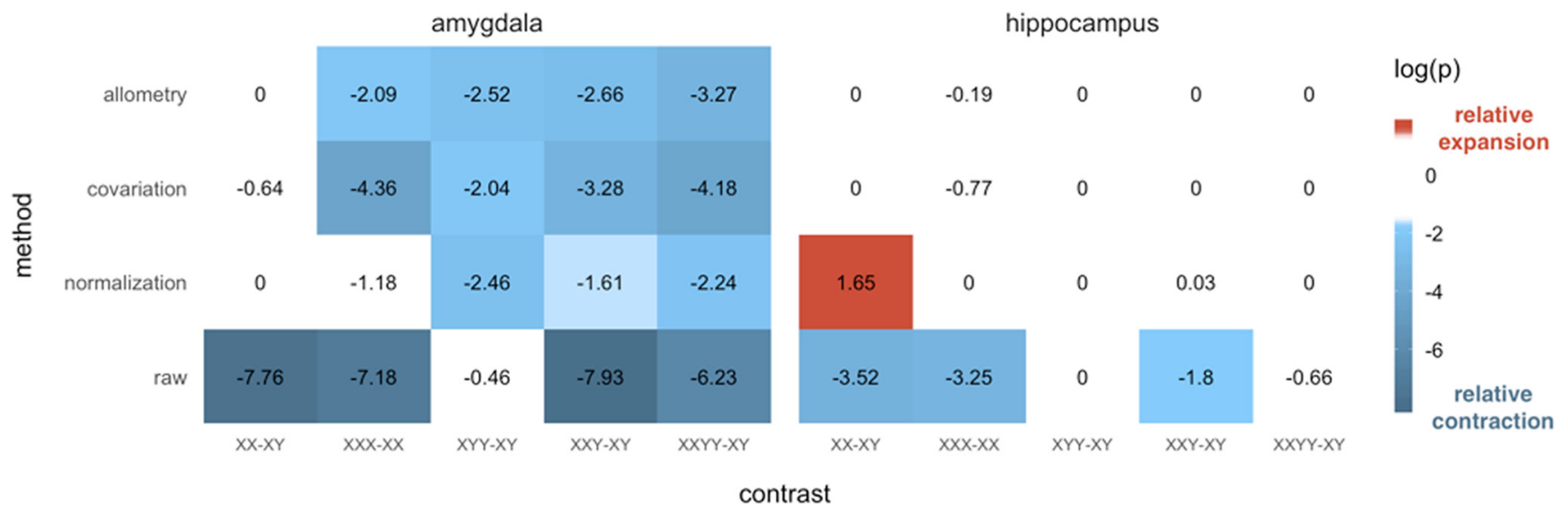

Figure 1. Bulk volume analysis of amygdala and hippocampus. (A) Total brain volume (tbv), amygdala, and hippocampus volume across karyotype groups, relative to typically developing males $(X Y)$. (B) Scaling of amygdala and hippocampus volume in the allometric sample (amygdala $\beta 1=0.89$; hippocampus $\beta 1=0.76$ ). (C) Comparisons of regional volume across sex and sex chromosome complement. FDR-corrected $\log (\mathrm{p})$ values are shown. Presence of color indicates statistical significance, and color identity/sign indicates direction of effect: for contrast $A-B$, blue and negative values indicate smaller volume in $A$ versus $B$, red and positive value indicate larger volume in A versus $B$.

were not included in this analysis due to missing Tanner stage data.

\section{Vertex-wise normative allometry of the amygdala and hippocampus}

To assess how sex and sex chromosome complement impact regional shape, we first developed a normative model for scaling of amygdala and hippocampus shape with size in our allometric sample. We found no significant interactive or additive effects of sex in our allometric model, leading us to use the simplest vertex-wise log-log regression between vertex surface area and total bilateral region surface area. We found that as total bilateral surface area of the amygdala and hippocampus increased, subregions scaled heterogeneously (Fig. 2A). For the amygdala, the rostral medial surface-scaled hyperallo- 


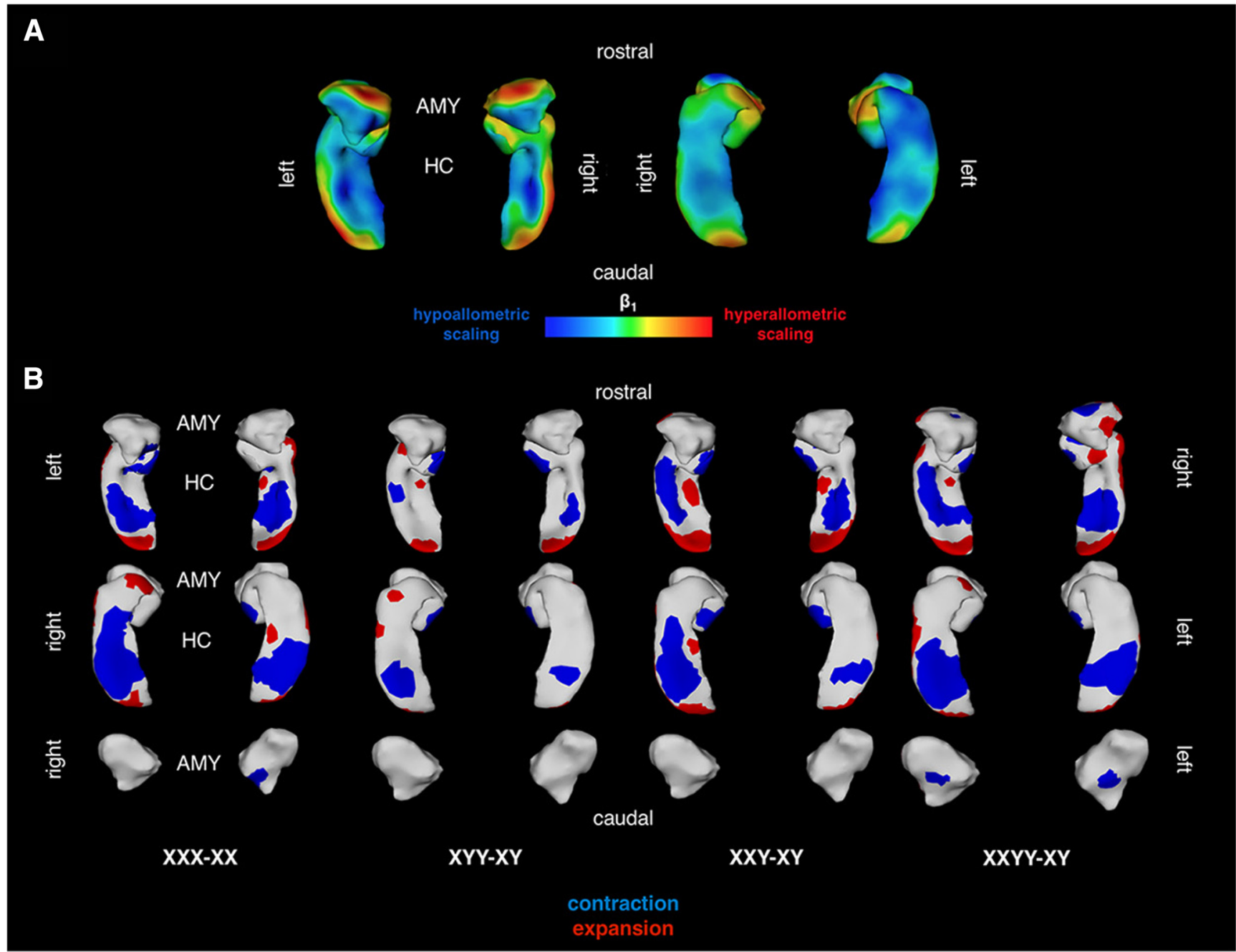

C

correspondence in spatial distribution of sex-chromosome effects (amygdala)

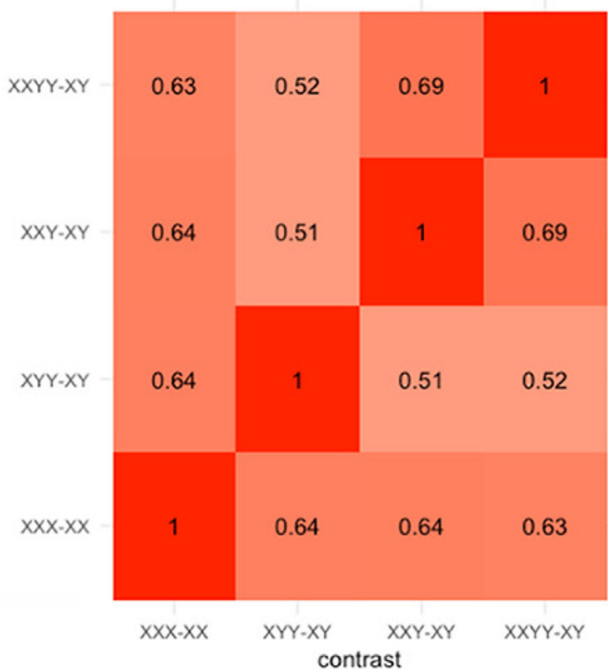

correspondence in spatial distribution of sex-chromosome effects (hippocampus)

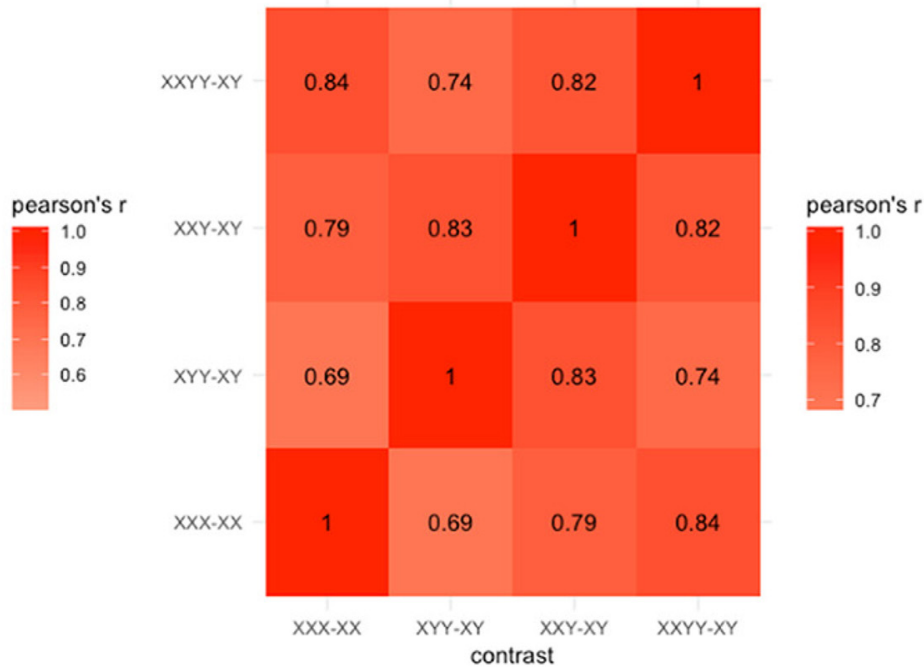

Figure 2. Surface-based analysis of amygdala and hippocampus shape. (A) Map of vertex-wise surface area scaling coefficients from log-log linear model with total bilateral region surface area. $(\boldsymbol{B})$ Binarized surface maps showing areas of significant surface area contraction (blue) or expansion (red) for each patient contrast. (C) Spatial correlation of uncorrected t-statistic maps demonstrating convergence of SCA effects. AMY: Amygdala, HC: Hippocampus. 
metrically, whereas the caudal lateral surface scaled hypoallometrically. For the hippocampus, the rostral and lateral surfaces scaled hyperallometrically, whereas the medial caudal surface scaled hypoallometrically.

\section{Amygdala shape is systematically altered by SCA}

Our normative description of amygdala scaling in health provided a foundation to assess whether amygdala shape is atypical in SCA. For each participant's scan, we computed vertex-wise deviations in surface area from normative scaling predictions based on total bilateral amygdala surface area, and asked whether these deviations differed across the karyotypic contrasts of interest.

After FDR correction, we observed statistically significant changes in proportional surface area in each patient group (Fig. 2B). In particular, we identified convergent changes in vertex-wise amygdala surface area across patient groups carrying a supernumerary $\mathrm{X}$-chromosome (Pearson's $r$ for inter-vertex variation in area change ranging from 0.63 to 0.69 ), and no significant differences in $\mathrm{XYY}$. However, when examining the spatial distribution of t-statistics before FDR correction, surface area changes in XYY were similar to surface area changes in the other three karyotype groups of interest (Pearson's $r$ ranging from 0.51 to 0.64 ; Fig. $2 C$ ). This result suggests that a supernumerary Y-chromosome exerts effects on amygdalar shape that are weaker than but nonetheless spatially convergent with effects of a supernumerary $\mathrm{X}$-chromosome. The general pattern of shape changes suggests that patches on the lateral surfaces of left and right amygdala are expanded in SCA (observed in XXY and $X X Y Y$ ), whereas patches on the ventral (XXX and $X X Y Y)$ and rostral (XXYY) surfaces are contracted in SCA. Covariation for age and tanner stage revealed additional significant vertices, including significantly contracting vertices on the ventral amygdala surface in $X X Y$ and $X Y Y$ that are concordant with contractions observed in $X X X$ and XXYY. Similar spatial correspondence was observed between different SCA effects after covariation for age and Tanner stage; a figure showing statistics for these comparisons is available online via FigShare (Nadig, 2018b). Notably, three XY participants, one XX participant, and one $X X Y$ participant included in the main analysis were not included in this analysis due to missing Tanner stage data.

\section{Hippocampal shape is systematically altered by SCA}

We applied a similar analysis strategy to the hippocampus surface to assess whether hippocampus shape is atypical in sex chromosome aneuploidies. After FDR correction, we observed a similar pattern of significant surface area difference to our amygdala analyses (Fig. 2B). In particular, we identified significant differences across $X$ aneuploidy groups relative to their respective gondal controls that were observable in attenuated form in the contrast between $X Y Y$ and $X Y$ groups (Pearson's $r$ ranging from 0.69 to 0.84 ; Fig. $2 C$ ). This pattern included bands of focal contraction along the ventral and dorsal surfaces of the hippocampus, and patches of focal expansion on the rostral extreme, caudal extreme, and medial portion of the ventral surface. Similar spatial correspondence was ob- served between different SCA effects after covariation for age and Tanner stage [a figure showing statistics for these comparisons is available online via FigShare (Nadig, 2018b)].

\section{Discussion}

Our analyses provide several insights into the influences of sex and sex chromosome complement on anatomic organization of the amygdala and hippocampus, as detailed below.

\section{Amygdala and hippocampus volume and shape are not sexually dimorphic}

Our findings replicate recent meta-analyses establishing that average amygdala and hippocampus volume are not statistically-significantly different between typically developing males and females after adjustment for sexdifferences in total brain volume (Tan et al., 2016; Marwha et al., 2017). In particular, although we observed large sex differences in raw amygdala and hippocampus volumes and an opposite effect in total-brain-volume-normalized hippocampal volume, these results were nonsignificant after both covariation- and allometry-based total brain volume adjustment. We conclude that gross differences in total brain volume between sexes, coupled to a hypoallometric scaling regime for the amygdala and hippocampus, may give rise to sex differences in raw and normalized region volumes that are secondary to total brain volume differences. We also used high-resolution surface-based shape assays to demonstrate that amygdala and hippocampus shape are also not significantly different between males and females. These results are in contrast to a previous report of sex differences in amygdala shape assessed via a different method (Kim et al., 2012), suggesting that choice of processing pipeline may influence detection of significant sex differences. Future studies should systematically assess consequences of processing pipeline choice to explain these disparate findings, as has been done for other subcortical compartments (Makowski et al., 2018). In light of recent evidence for publication bias in neuroimaging of sex differences, our null findings are an important contribution to a body of literature that generally overestimates sex differences in the human brain (David et al., 2018). Thus, well established sex-biases in the prevalence rates of psychiatric disorders that are linked to the amygdala and hippocampus do not appear to be accompanied by clear sex-biases in the macroanatomical organization of these structures in a typically developing population.

\section{Carriage of supernumerary sex chromosomes changes proportional amygdala volume, but not proportional hippocampus volume}

We found that supernumerary sex chromosomes decrease the proportional volume of the amygdala. This decrease was common across aneuploidy groups, and mostly consistent across techniques to account for total brain size. The exception to this generally observed de-

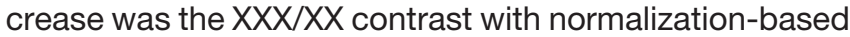
adjustment for total brain volume, where no significant difference in amygdala volume was detected. Converging 
evidence across other total brain volume adjustment techniques and related patient groups suggests that this null finding may arise from methodological issues with normalization by division. Future simulation studies should assess why normalization by division yields different results, and in what situations it is or is not an appropriate technique to control for total brain volume. This amygdala volume decrease in patients carrying supernumerary sex chromosomes is consistent with a previously observed amygdala volume increase in patients with X-chromosome monosomy (Turner's syndrome; Kesler et al., 2004). These results suggest that amygdala volume is negatively correlated with sex chromosome dosage, and indicate that the increases in both $\mathrm{X}$ - and $\mathrm{Y}$-chromosome dosage can induce disproportionate reductions in amygdala volume relative to their effects on overall brain size. Although we detected differences in raw hippocampal volume in $X X X$ females and $X X Y$ males relative to controls, these effects were not significant after total brain volume adjustment, suggesting that raw volume changes may be secondary to changes in total brain volume.

\section{Carriage of supernumerary sex chromosome alters amygdala and hippocampus shape}

Carriage of additional X- and Y-chromosomes were both associated with a similar topography of shape change in the amygdala and hippocampus, although visual examination of FDR-corrected maps (Fig. 2B) show clearly that the extent of significant effects was greater in $X$ - versus $Y$-chromosome aneuploidy. In particular, an area along the lateral surfaces of the amygdala was expanded in XXY and XXYY, patches on the ventral surface of the amygdala were contracted in $X X X$ and $X X Y Y$, and an area centered on the basomedial nucleus was focally contracted in XXYY. Our report of spatially specific contraction of the basomedial nucleus in XXYY, in combination with a previous report of spatially specific expansion of the basomedial nucleus in X-chromosome monosomy (Green et al., 2016), suggests a spatially specific $X$-chromosome-dosage effect on amygdala shape, although this effect was not statistically significant in XXX or $X X Y$. In the hippocampus, we observed a large band of areal contraction along the midsection of the rostralcaudal axis, as well as small areas of focal expansion at the rostral extreme, caudal extreme, and medial portion on the ventral surface of the hippocampus. These areas of shape change cut across hippocampal subfields, suggesting that SCA does not target particular hippocampal compartments. Importantly, we show that SCA effects in amygdala and hippocampus shape are not a simple byproduct of changes in the overall size of these structures. Future studies should confirm our findings with high-field MRI images, and align observed shape changes to known functional, connectivity, and cytoarchitectonic gradients within the amygdala and hippocampus. These studies would ideally identify core subregional modules of vulnerability to copy number variation that explain elevated rates of psychiatric disorders seen in SCA (Hong and Reiss, 2014).

\section{Conclusions}

Carriage of supernumerary $\mathrm{X}$ - and $\mathrm{Y}$ - chromosomes exert convergent effects on the volume and shape of the amygdala and hippocampus. This convergence across the entire family of SCAs echoes convergent effects previously identified in the cerebral cortex (Raznahan et al., 2016), striatum and thalamus (Reardon et al., 2016), and cerebellum (Mankiw et al., 2017). This robust pattern suggests that SCA influence on brain anatomy arises from the shared gene content between $\mathrm{X}$ - and $\mathrm{Y}$-chromosomes [i.e., pseudoautosomal regions (PARs) or $X / Y$ gametolog genes]. Indeed, recent in vitro gene expression results have identified a tendency for such shared genes to show robust differential expression in SCA (Raznahan et al., 2018).

These findings extend upon existing literature examining neuroanatomy in SCA patients in three key ways. First, our results are the first characterization of limbic anatomy changes in $47 \mathrm{XYY}$ patients. The finding of reduced amygdala volume in these patients may be relevant for the observation that $\mathrm{Y}$-chromosome aneuploidy is often accompanied by social deficits and autistic symptoms (Lee et al., 2012), given well documented patterns of amygdala changes in autistic patients (Nordahl et al., 2012; Avino et al., 2018). Second, the present findings highlight the power of a comparative approach that profiles brain phenotypes across a family of related disorders, rather than for single disorders in isolation from each other this approach has identified robust biomarkers across a range of brain disorders and imaging assays (Goodkind et al., 2015; Kaczkurkin et al., 2017; Spronk et al., 2018). This approach helps identify convergent effects across many genetic insults and reinforces reproducibility of findings across variation in genetic copy number. Third, our results demonstrate how high-resolution surface analyses can complement traditional bulk volume analyses. In particular, although SCA was not accompanied by a change in hippocampal volume, our surface-based analysis identified a robust, spatially consistent change in hippocampus shape that may have consequences for the function of neuronal circuits therein.

The present study has four key limitations. First, we do not address behavioral correlates of the observed changes in limbic anatomy; performing such analyses would be critical to fully assess whether limbic anatomy is a biomarker for phenotypic severity. Second, because our sample is cross-sectional, we cannot assess how observed effects are patterned across developmental time within individuals. Although longitudinal studies are difficult in rare patient populations, these data would help clarify the temporal properties of limbic anatomy development in SCA. Third, because some SCAs are accompanied by deficits in gonadal function (Hong and Reiss, 2014), we cannot unambiguously attribute observed changes in amygdala and hippocampus structure to sex chromosome aneuploidy rather than differences in circulating gonadal hormones. However, we observe consistent anatomic effects of a supernumerary $\mathrm{X}$-chromosome 
across patient groups with noted gonadal deficits accompanying the extra X-chromosome (e.g., XXYY, XXY) and patient groups without any such accompanying gonadal deficits (e.g., XXX), suggesting that observed effects arise from sex chromosomes rather than gonadal pathology. Last, although structural MRI is a powerful tool for assessing gross regional volume and shape, it does not allow direct embedding of our present findings within known microarchitectural gradients of the limbic system (Janak and Tye, 2015).

Notwithstanding these limitations, our study provides a step toward understanding how copy number variation shapes the brain systems that underlie risk for psychopathology and, more broadly, social and emotional life. Understanding how a range of SCAs cause psychopathology via limbic brain systems may help triangulate stereotyped risk pathways that can serve as biomarkers and targets for intervention.

\section{References}

Ad-Dab'bagh Y, Lyttelton O, Muehlboeck JS, Lepage C, Einarson D, Mok K, Ivanov O, Vincent RD, Lerch J, Fombonne E, Evans AC (2006) The CIVET image-processing environment: a fully automated comprehensive pipeline for anatomical neuroimaging research. In Proceedings of the 12th annual meeting of the organization for human brain mapping (2266). Florence, Italy.

Amaral DG, Schumann CM, Nordahl CW (2008) Neuroanatomy of autism. Trends Neurosci 31:137-145. CrossRef Medline

Avino TA, Barger N, Vargas MV, Carlson EL, Amaral DG, Bauman MD, Schumann CM (2018) Neuron numbers increase in the human amygdala from birth to adulthood, but not in autism. Proc Natl Acad Sci U S A 115:3710-3715. CrossRef

Carr VA, Rissman J, Wagner AD (2010) Imaging the human medial temporal lobe with high-resolution fMRI. Neuron 65:298-308. CrossRef Medline

Chakravarty MM, Steadman P, van Eede MC, Calcott RD, Gu V, Shaw P, Raznahan A, Collins DL, Lerch JP (2013) Performing label-fusion-based segmentation using multiple automatically generated templates. Hum Brain Mapp 34:2635-2654. CrossRef

Cocosco CA, Zijdenbos AP, Evans AC (2003) A fully automatic and robust brain $\mathrm{MRI}$ tissue classification method. Med Image Anal 7:513-527. CrossRef

David SP, Naudet F, Laude J, Radua J, Fusar-Poli P, Chu I, Stefanick ML, loannidis JPA (2018) Potential reporting bias in neuroimaging studies of sex differences. Sci Rep 8:6082.

Fish AM, Cachia A, Fischer C, Mankiw C, Reardon PK, Clasen LS, Blumenthal JD, Greenstein D, Giedd JN, Mangin JF, Raznahan A (2016) Influences of brain size, sex, and sex chromosome complement on the architecture of human cortical folding. Cerebral Cortex 27: 5557-5567. CrossRef Medline

Giedd JN, Raznahan A, Alexander-Bloch A, Schmitt E, Gogtay N, Rapoport JL (2015) Child psychiatry branch of the National Institute of Mental Health longitudinal structural magnetic resonance imaging study of human brain development. Neuropsychopharmacology 40:43-49. CrossRef

Goodkind M, Eickhoff SB, Oathes DJ, Jiang Y, Chang A, JonesHagata LB, Ortega BN, Zaiko YV, Roach EL, Korgaonkar MS, Grieve SM, Galatzer-Levy I, Fox PT, Etkin A (2015) Identification of a common neurobiological substrate for mental illness. JAMA Psychiatry 72:305-315. CrossRef

Green T, Fierro KC, Raman MM, Foland-Ross L, Hong DS, Reiss AL (2016) Sex differences in amygdala shape: insights from Turner syndrome. Hum Brain Mapp 37:1593-1601. CrossRef

Hennessey T, Andari E, Rainnie DG (2018) RDoC-based categorization of amygdala functions and its implications in autism. Neurosci Biobehav Rev 90:115-129. CrossRef
Hong DS, Reiss AL (2014) Cognitive and neurological aspects of sex chromosome aneuploidies. Lancet Neurol 13:306-318. CrossRef Medline

Huxley JS (1924) Constant differential growth-ratios and their significance. Nature 114:895-896. CrossRef

Janak PH, Tye KM (2015) From circuits to behaviour in the amygdala. Nature 517:284-292. CrossRef Medline

Kaczkurkin AN, Moore TM, Calkins ME, Ciric R, Detre JA, Elliott MA, Garcia de la Garza A, Roalf DR, Rosen A, Ruparel K, Shinohara RT, Xia CH, Wolf DH, Gur RE, Gur RC, Satterthwaite TD (2017) Common and dissociable regional cerebral blood flow differences associate with dimensions of psychopathology across categorical diagnoses. Mol Psychiatry. Advance online publication. doi: 10.1038/mp.2017.174.

Kesler SR, Garrett A, Bender B, Yankowitz J, Zeng SM, Reiss AL (2004) Amygdala and hippocampal volumes in Turner syndrome: a high-resolution MRI study of X-monosomy. Neuropsychologia 42: 1971-1978. CrossRef

Kim HJ, Kim N, Kim S, Hong S, Park K, Lim S, Park JM, Na B, Chae Y, Lee J, Yeo S, Choe IH, Cho SY, Cho G (2012) Sex differences in amygdala subregions: evidence from subregional shape analysis. Neurolmage 60:2054-2061. CrossRef Medline

Lee NR, Wallace GL, Adeyemi El, Lopez KC, Blumenthal JD, Clasen LS, Giedd JN (2012) Dosage effects of $X$ and $Y$ chromosomes on language and social functioning in children with supernumerary sex chromosome aneuploidies: implications for idiopathic language impairment and autism spectrum disorders. J Child Psychol Psychiatry 53:1072-1081. CrossRef

Lin A, Clasen L, Lee NR, Wallace GL, Lalonde F, Blumenthal J, Giedd JN, Raznahan A (2015) Mapping the stability of human brain asymmetry across five sex-chromosome aneuploidies. Journal of Neuroscience 35:140-145. CrossRef Medline

Makowski C, Béland S, Kostopoulos P, Bhagwat N, Devenyi GA, Malla AK, Joober R, Lepage M, Chakravarty MM (2018) Evaluating accuracy of striatal, pallidal, and thalamic segmentation methods: Comparing automated approaches to manual delineation. NeuroImage 170:182-198. CrossRef

Mankiw C, Park MTM, Reardon PK, Fish AM, Clasen LS, Greenstein D, Giedd JN, Blumenthal JD, Lerch JP, Chakravarty MM, Raznahan A (2017) Allometric analysis detects brain size-independent effects of sex and sex chromosome complement on human cerebellar organization. J Neurosci 37:5221-5231. CrossRef

Marwha D, Halari M, Eliot L (2017) Meta-analysis reveals a lack of sexual dimorphism in human amygdala volume. Neurolmage 147: 282-294. CrossRef Medline

Nordahl CW, Scholz R, Yang X, Buonocore MH, Simon T, Rogers S, Amaral DG (2012) Increased rate of amygdala growth in children aged 2 to 4 years with autism spectrum disorders: a longitudinal study. Arch Gen Psychiatry 69:53-61. CrossRef

Nadig A (2018a). Results of pairwise volumetric contrasts across sex and sex chromosome aneuploidy after covariation for age and Tanner stage (Version 1). figshare. doi: 10.6084/m9.figshare.6981854.v1.

Nadig A (2018b). Results of pairwise surface-based shape contrasts across sex chromosome aneuploidy groups after covariation for age and Tanner stage (Version 1). figshare. doi: 10.6084/ m9.figshare.6990008.v1.

Pendersen TL (2017). patchwork: the Composer of ggplots (Version $\mathrm{R}$ package version 0.0.1). Retrieved from https://github.com/ thomasp85/patchwork.

Pipitone J, Park MTM, Winterburn J, Lett TA, Lerch JP, Pruessner JC, Lepage M, Voineskos AN, Chakravarty MM; Alzheimer's Disease Neuroimaging Initiative (2014) Multi-atlas segmentation of the whole hippocampus and subfields using multiple automatically generated templates. Neurolmage 101:494-512. CrossRef

Price JL, Drevets WC (2012) Neural circuits underlying the pathophysiology of mood disorders. Trends Cogn Sci 16:61-71. CrossRef Medline 
Raznahan A, Lee NR, Greenstein D, Wallace GL, Blumenthal JD, Clasen LS, Giedd JN (2016) Globally divergent but locally convergent $\mathrm{X}$ - and $\mathrm{Y}$-chromosome influences on cortical development. Cereb Cortex 26:70-79. CrossRef

Raznahan A, Parikshak N, Chandran V, Blumenthal J, Clasen L, Alexander-Bloch A, Zinn AR, Wangsa D, Wise J, Murphy DG, Bolton PF, Ried T, Ross J, Giedd JN, Geschwind D (2018) Sex chromosome dosage effects on gene expression in humans. Proc Natl Acad Sci U S A 115:7398-7403. CrossRef Medline

R Core Team (2008) R: a language and environment for statistical computing. Vienna, Austria; R Foundation for Statistical Computing. Retrieved from https://www.R-project.org/

Reardon PK, Clasen L, Giedd JN, Blumenthal J, Lerch JP, Chakravarty MM, Raznahan A (2016) An allometric analysis of sex and sex chromosome dosage effects on subcortical anatomy in humans. $J$ Neurosci 36:2438-2448. CrossRef

Reardon PK, Seidlitz J, Vandekar S, Liu S, Patel R, Park MT, Alexander-Bloch A, Clasen LS, Blumenthal JD, Lalonde FM, Giedd JN, Gur RC, Gur RE, Lerch JP, Chakravarty MM, Satterthwaite TD, Shinohara RT, Raznahan A (2018) Normative brain size variation and brain shape diversity in humans. Science 360:1222-1227. CrossRef Medline

Ruscio AM, Hallion LS, Lim CCW, Aguilar-Gaxiola S, Al-Hamzawi A, Alonso J, ... Scott KM (2017) Cross-sectional comparison of the epidemiology of dsm- 5 generalized anxiety disorder across the globe. JAMA Psychiatry 74:465-475. CrossRef

Saygin ZM, Osher DE, Koldewyn K, Martin RE, Finn A, Saxe R, Gabrieli JD, Sheridan M (2015) Structural connectivity of the developing human amygdala. PloS One 10:e0125170. CrossRef Medline

Spronk M, Kulkarni K, Ji JL, Keane B, Anticevic A, Cole MW (2018) $A$ whole-brain and cross-diagnostic perspective on functional brain network dysfunction. bioRxiv 326728. Advance online publication. doi: 10.1101/326728

Tan A, Ma W, Vira A, Marwha D, Eliot L (2016) The human hippocampus is not sexually-dimorphic: meta-analysis of structural MRI volumes. Neurolmage 124:350-366. CrossRef Medline

Werling DM, Geschwind DH (2013) Sex differences in autism spectrum disorders. Curr Opin Neurol 26:146-153. CrossRef Medline

Wickham H (2016) ggplot2: elegant graphics for data analysis. Berlin, Germany: Springer.

Winterburn JL, Pruessner JC, Chavez S, Schira MM, Lobaugh NJ, Voineskos AN, Chakravarty MM (2013) A novel in vivo atlas of human hippocampal subfields using high-resolution $3 \mathrm{~T}$ magnetic resonance imaging. Neurolmage 74:254-265. CrossRef

Zijdenbos AP, Forghani R, Evans AC (2002) Automatic "pipeline" analysis of 3-D MRI data for clinical trials: application to multiple sclerosis. IEEE Trans Med Imag 21:1280-1291. CrossRef 